\title{
Inferring Aggressive Driving Behavior from Smartphone Data - Smartphone's sensors meet Inception
}

\author{
Anonymous Author(s) \\ Affiliation \\ Address \\ email
}

\section{Motivation}

Road accidents occur all over the world. The World Health Organization (WHO) estimates that every year approximately 1.35 million people die and between 20 to 50 million people suffer injuries as a result of road traffic crashes[1]. The causes of these fatalities are varied, but the AAA Foundation for Traffic Safety has found that more than a half of the fatal accidents that occurred between 2003 and 2007 (just in the U.S.) were related to aggressive driving behavior [2]. To mitigate these problems, we need to raise awareness of aggressive driving behavior and provide driving feedback on an individual level.

This study aims to exploit driving data collected from sensors embedded in our own smartphones. In this way, the smartphone can be made to act as our copilot and make us aware of risky decisions that we make behind the driving wheel. By using smartphones rather than, for example, a) Aftermarket systems [3, 4], b) On Board Diagnostics (OBD) systems [3][5][6] and, c) Vehicle-mounted video cameras [7][8], we achieve broad applicability of this study by allowing practically any driver in any car to be supported by this technology. As we know, smartphones come integrated with a variety of sensors, some of which (accelerometer, gyroscope, magnetometer) can be used to measure accelerations and forces that the smartphone itself experiences [9]. There are several reports in the literature where they following this approach of analyzing, via Machine Learning pipelines, all available smartphone sensor data to infer the driving action that might have caused it [10, 11, 7, 5, 12, 13]. This is known as Maneuver Classification. By solving this classification problem we can then attempt to generate a more informative Driving behavior profile, which could be used to measure improvements against oneself or within a community of friends under a gamification scenario, or even to make profit of this information by reducing insurance premiums [14].

\section{Problem Statement}

The processing of smartphone sensor data is not trivial, the signal-to-noise ratio is commonly low. A common approach to deal with this problem is to use only one sensor at a time to reduce information overload [11], then evaluating which sensor has better capabilities to detect certain driving maneuvers. On the other hand, there are studies where the data from different sensors is concatenated within the same feature vector [15]. This then enables a multitude of discriminative algorithms to relate the data to specific phenomena. In this study, we attempt to improve how this learning problem is approached by means of two original ideas:

- Design a Gated mechanism able to learn to discriminate what sensor data to use and what to ignore, then efficiently balancing the available sensor data

- Exploit the native structure of sensor data, which is a time series, by means of ad-hoc state-of-the-art approaches such as the InceptionTIME Neural Network 


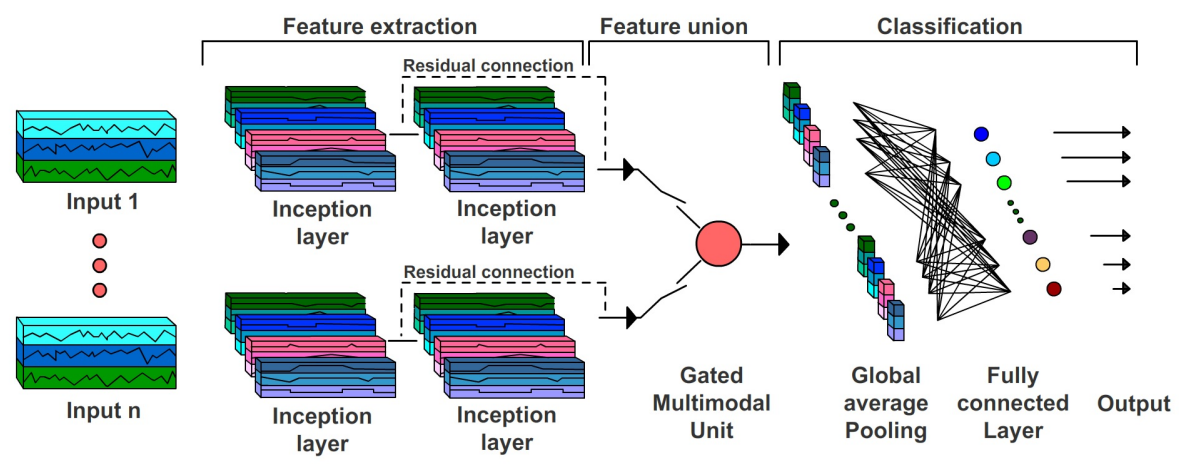

Figure 1: Proposed model with 2 inceptions layers and a Gated multimodal unit.
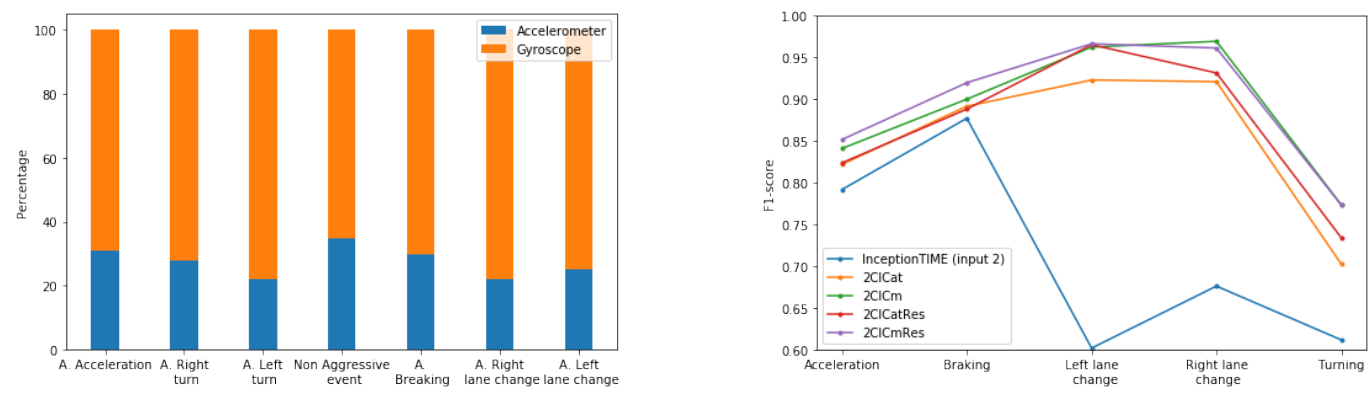

Figure 2: (a)Gated multimodal units performance; (b) Comparison of different models on several driving maneuvers

Both of these innovations are integrated within the same deep architecture. The intuition behind this workflow is based on the success that InceptionTime[16] has showed as a state-of-the-art method to classify generic time series, and also on results that show that Gated Multimodal Units [17] improve classification performance by finding intermediate representations based on data from different modalities.

\section{Experiments and Results}

For illustration purposes, Figure 1 shows a schematic proposal of the integration of the ideas just stated. For the evaluation of these architectures we selected two state-of-the-art datasets used to detect driver's aggressive maneuvers: Ferreira dataset [11] and the UAH-dataset [18]. As we have stated, the literature has not envisioned mechanisms to explicitly discriminate parts of or all sensor data that is useless for classification. When this model receives Accelerometer and Gyroscope data as input it learns to weight how much importance it needs to pay to each data source (similar to an Attention mechanism!); this phenomenon can be observed in Figure 2(a). As can be seen, the gated unit learns the specific amount of source data to flow through it for each individual maneuver (x-axis). As expected, turning and lane changing events, that is, events involving some kind of rotation, rely more on gyroscopes than braking and acceleration events. The next experiment deals with classification performance of different models based on the $F_{1}$ score. We evaluate several variations of models that incorporate InceptionTIME and gated units on the two aforementioned datasets. In this study we will show the results on the UAH-dataset. Figure 2(b) presents a comparison among different architecture proposals (all starting with code name "2Cl") against the original and robust InceptionTIME network. It can be observed that all the proposed models produce very competitive performance, and in all cases outperforming InceptionTIME. At this point, we are focused on reducing the computational complexity of these models to allow them to be executed on regular smartphones. 


\section{References}

[1] "Road traffic injuries," feb 2020. [Online]. Available: https://www.who.int/news-room/ fact-sheets/detail/road-traffic-injuries

[2] AAAFoundation, “Aggressive Driving : Research Update," AAA Foundation for Traffic Safety, Washington DC, Tech. Rep., 2009.

[3] B. Jachimczyk, D. Dziak, J. Czapla, P. Damps, and W. Kulesza, "Iot on-board system for driving style assessment," Sensors, vol. 18, p. 1233, 042018.

[4] S. G. Christopoulos, S. Kanarachos, and A. Chroneos, "Learning driver braking behavior using smartphones, neural networks and the sliding correlation coefficient: Road anomaly case study," IEEE Transactions on Intelligent Transportation Systems, vol. 20, no. 1, pp. 65-74, Jan 2019.

[5] J. Zhang, Z. Wu, F. Li, C. Xie, T. Ren, C. Jie, and L. Liu, "A deep learning framework for driving behavior identification on in-vehicle can-bus sensor data," Sensors, vol. 19, p. 1356, 03 2019.

[6] M. Shahverdy, M. Fathy, R. Berangi, and M. Sabokrou, "Driver behavior detection and classification using deep convolutional neural networks," Expert Systems with Applications, vol. 149, p. 113240, 2020. [Online]. Available: http://www.sciencedirect.com/science/article/pii/ S095741742030066X

[7] Y. Moukafih, H. Hafidi, and M. Ghogho, "Aggressive driving detection using deep learningbased time series classification," in 2019 IEEE International Symposium on INnovations in Intelligent SysTems and Applications (INISTA), July 2019, pp. 1-5.

[8] M. Karaduman and H. Eren, "Deep learning based traffic direction sign detection and determining driving style," in 2017 International Conference on Computer Science and Engineering (UBMK), Oct 2017, pp. 1046-1050.

[9] M. R. Carlos, L. C. González, J. Wahlström, G. Ramírez, F. Martínez, and G. Runger, "How smartphone accelerometers reveal aggressive driving behavior?- the key is the representation," IEEE Transactions on Intelligent Transportation Systems, vol. 21, no. 8, pp. 3377-3387, 2020.

[10] M. M. Bejani and M. Ghatee, "Convolutional Neural Network With Adaptive Regularization to Classify Driving Styles on Smartphones," IEEE Transactions on Intelligent Transportation Systems, pp. 1-10, 2019.

[11] J. Ferreira Júnior, E. Carvalho, B. V. Ferreira, C. de Souza, Y. Suhara, A. Pentland, and G. Pessin, "Driver behavior profiling: An investigation with different smartphone sensors and machine learning," PLOS ONE, vol. 12, no. 4, pp. 1-16, 2017. [Online]. Available: https://doi.org/10.1371/journal.pone.0174959

[12] J. Guo, Y. Liu, L. Zhang, and Y. Wang, "Driving behaviour style study with a hybrid deep learning framework based on gps data,’ Sustainability, vol. 10, p. 2351, 072018.

[13] J. Zhang, Z. Wu, F. Li, J. Luo, T. Ren, S. Hu, W. Li, and W. Li, "Attention-based convolutional and recurrent neural networks for driving behavior recognition using smartphone sensor data," IEEE Access, pp. 1-1, 2019.

[14] J. R. López, L. C. González, J. Wahlström, M. Montes y Gómez, L. Trujillo, and G. RamírezAlonso, "A genetic programming approach for driving score calculation in the context of intelligent transportation systems," IEEE Sensors Journal, vol. 18, no. 17, pp. 7183-7192, 2018.

[15] D. A. Johnson and M. M. Trivedi, "Driving style recognition using a smartphone as a sensor platform," in 2011 14th International IEEE Conference on Intelligent Transportation Systems (ITSC), Oct 2011, pp. 1609-1615.

[16] H. Ismail Fawaz, B. Lucas, G. Forestier, C. Pelletier, D. Schmidt, J. Weber, G. Webb, L. Idoumghar, P.-A. Muller, and F. Petitjean, "InceptionTime: Finding AlexNet for Time Series Classification," 2019.

[17] J. Arevalo, T. Solorio, M. Montes, and F. González, "Gated Multimodal Units for Information Fusion," 2017.

[18] E. Romera, L. M. Bergasa, and R. Arroyo, "Need data for driver behaviour analysis? Presenting the public UAH-DriveSet," in 2016 IEEE 19th International Conference on Intelligent Transportation Systems (ITSC), nov 2016, pp. 387-392. 